\title{
Cuidados odontológicos no atendimento domiciliar
}

\author{
Dental care in home care \\ Cuidado dental en el cuidado domiciliario
}

José Milton de Aquino e Silva Neto ${ }^{1 *}$, Tatianne Michelle Delgado do Nascimento ${ }^{1}$, Alessandra Soares da Silva ${ }^{1}$, Camilla Lins dos Anjos ${ }^{1}$, Izabel Cristina Gomes de Mendonça1.

\section{RESUMO}

Objetivo: Este trabalho tem como objetivo evidenciar os principais aspectos da visita domiciliar em saúde bucal na atenção primária. Revisão bibliográfica: A incorporação do atendimento domiciliar aponta para uma reorganização das práticas de saúde além dos muros dos serviços de saúde, deslocando o olhar crítico para um espaço em que o paciente vive sendo enquadrado nesse aspecto, tanto o usuário, quanto a família e a comunidade nos quais as práticas das atenções estão enraizadas, identificando as particularidades, e quais seriam aquelas famílias que de alguma forma precisam de um maior acesso a porta de entrada no Sistema Único de Saúde (SUS). A visita domiciliar atende aos princípios norteadores das ações especificas nas diretrizes da Política nacional de Saúde Bucal, bem como os princípios da ética da saúde, o acesso universal para a assistência e o acolhimento, por isso é de suma importância o atendimento com uma equipe multidisciplinar incluindo o Cirurgião Dentista $(C D)$. Considerações finais: $O$ atendimento domiciliar não é implantado em todos os territórios no sentido das atividades e estratégias de saúde bucal, porém vale ressaltar que o CD deve ser inserido buscando cuidar de maneira integral da cavidade bucal dos pacientes.

Palavras-chave: Saúde bucal, Clínicas odontológicas, Saúde da família, Visita domiciliar.

\begin{abstract}
Objective: This study aims to highlight the main aspects of home visits in oral health in primary care. Revisión bibliográfica: The incorporation of home care points to a reorganization of health practices beyond the walls of health services, shifting the critical eye to a space in which the patient lives being framed in this aspect, both the user, the family and the community in which they live. the practices of care are rooted, identifying the particularities, and which would be those families that somehow need greater access to the gateway to the Unified Health System (SUS). The home visit meets the guiding principles of the specific actions in the guidelines of the National Oral Health Policy, as well as the principles of health ethics, universal access for assistance and reception, so it is extremely important to attend with a multidisciplinary team including the Dental Surgeon (CD). Final considerations: Home care is not implemented in all territories in terms of oral health activities and strategies, however it is worth mentioning that the DC should be inserted in order to fully care for patients' oral cavity.
\end{abstract}

Keywords: Oral health, Dental clinics, Family health, House calls.

\section{RESUMEN}

Objetivo: Este estudio tiene como objetivo destacar los principales aspectos de las visitas domiciliarias en salud bucodental en atención primaria. Literature review: La incorporación de la atención domiciliaria apunta a una reorganización de las prácticas de salud más allá de los muros de los servicios de salud, desplazando la mirada crítica hacia un espacio en el que vive el paciente enmarcándose en este aspecto, tanto el usuario, la familia y la comunidad en la que vive. Se arraigan las prácticas de atención, identificando las particularidades, y cuáles serían aquellas familias que de alguna manera necesitan mayor acceso a la puerta de entrada al Sistema Único de Salud (SUS). La visita domiciliaria cumple con los principios rectores de las acciones específicas en los lineamientos de la Política Nacional de Salud Bucal, así como con los principios de ética en salud, acceso universal para la atención y recepción, por lo que es de suma importancia atender con un equipo multidisciplinario que incluya al Cirujano dentista (CD). Consideraciones finales: La atención domiciliaria no está implementada en todos los territorios en cuanto a actividades y estrategias de salud bucal, sin embargo, cabe mencionar que la CD debe ser insertada para poder cuidar integralmente la cavidad bucal de los pacientes.

Palabras clave: Salud bucal, Clínicas odontológicas, Salud de la familia, Visita domiciliaria.

${ }^{1}$ Centro Universitário CESMAC (CESMAC), Maceió - AL. *E-mail: milton_neto_166@hotmail.com 


\section{INTRODUÇÃO}

A criação do Sistema Único de Saúde (SUS) veio a ser desencadeado com o objetivo de ser um padrão regulamentador do qual buscava ser uma inovação ao restabelecer uma nova organização socioeconômica, no entanto, esse novo modelo sofreu alguns impactos devido o processo histórico presente no Brasil, sendo vivido por pessoas que eram reprimidas, ou seja, não apresentavam um poder aquisitivo legal, com uma desigualdade extrema e não apresentavam cuidados de atenção básica no decorrer de sua vida. Com o surgimento do modelo assistencialista referente ao SUS, a realidade dessas pessoas foi modificada, tendo como principal foco beneficiar a todos com a universalidade, integralidade e equidade, com esse desenvolvimento todos os seres humanos tiveram direito a saúde de maneira mais humanizada (REIS WG, et al., 2015; SILVA RM, et al., 2018).

Com a criação da portaria 2529 de 19 de outubro de 2006 que tinha como instituir a Internação Domiciliar no âmbito do SUS, dos quais definia a atenção domiciliar no âmbito do SUS, não especificando alguma patologia ou algum nível de atenção que o usuário poderia ter, mas embasou-se na atenção domiciliar (AD) enquanto uma política de Governo de Estado voltada para a construção realmente de uma equipe de AD e fomento à serviço dessas atenções, infelizmente essa portaria não gerou a criação dessas equipes, e com a nova portaria 2527, de 27 de outubro de 2011 redefiniu a AD no âmbito do SUS classificando suas atenções, com isso foram criados através dessa portaria três modelos de trabalho, sendo distinguido pelo nível 1,2 e 3 (ROCHA DA e FRANCO MA, 2013; MIRANDA AF, et al., 2018).

Posteriormente surgiu a portaria 963, de 27 de maio de 2013, dos quais redefine a atenção domiciliar no âmbito do Sistema Único de Saúde, enquanto a portaria 2527 de 27 de outubro de 2011, previa uma equipe de AD para cada 100 mil habitantes a posteriormente em 2013 a previsão caiu para 20 mil habitantes com a possibilidade dos municípios se consociarem. Só que com essas condições surgiram as seguintes situações, se for levado em consideração, o máximo de cobertura que essas equipes poderiam atuar seria apenas metade da população brasileira, ou seja, a outra metade não seria (BRASIL, 2013).

Com isso observa-se que justamente aquela população que por possuir moradia em um município de menor porte não iria usufruir desses cuidados devido a pequena quantidade de habitantes, não se beneficiando assim para poder montar sua equipe de $A D$, além disso existe uma segunda situação, que é a presença das equipes de $A D$ no município, porém a necessidade da inter-relação com os cuidados realizados pelas equipes de $A B$ (BRASIL, 2013).

O caderno que corresponde a Atenção Básica no 17, orienta a maneira correta de atenção à saúde bucal no SUS, destaca que a constituição da AD é imprescindível, contudo detém certos empecilhos quando se alega a Estratégia da saúde Básica (ESB) (BRASIL, 2006). A AD relacionada a saúde inclui de maneira direta as visitas domiciliares aos usuários que têm necessidade de possuir assistências básicas de saúde, como pode ser citado os suportes assistenciais e as internações domiciliares, estabelecendo cada um com distintos cuidados (REIS WG, et al., 2015).

Com a nova regularização foi implantado a visita domiciliar, dos quais observou que apresenta grande importância no âmbito da educação das pessoas e nas prevenções de inúmeras patologias, e ela possibilita o conhecimento da saúde da família de determinado território com maior profundidade, sendo estabelecidos desenvolvimentos de ações promocionais e o acompanhamento de grupos, sendo constituída em forma de elementos no estabelecimento de vínculos entre as equipes e as famílias. Na saúde bucal ela não foca necessariamente apenas na educação preventiva, podendo ser feito intervenções em algumas situações no leito ou na residência de usuários que são mais restritos ao seu acompanhamento, através do programa de assistência domiciliar (PINTO VG, 2013; ROCHA DA e FRANCO MA, 2013).

A vista desses pontos, o papel da visita domiciliar, baseia-se em conseguir ampliar o acesso dos usuários, nos serviços ofertados pelo SUS, tendo em vista que muitos possuem dificuldades de locomoção ou estão restritos ao leito, mas a visita domiciliar não enquadra-se só a pessoas que possuem dificuldades de acessos, em alguns casos sãos levado em consideração grupos de famílias que apresentam vulnerabilidade ou questões que são de ações programadas, a VD pode ser uma boa estratégia, para conseguir acessar as 
pessoas, alguns exemplos onde essas questões são discutidas e evidenciadas podem ser: na análise da saúde das gestantes, buscas ativa de crianças, pessoas hipertensas, diabéticos e obesos (BIZERRIL DO, et al., 2015; ANDRÉ PL, et al., 2019).

Mediante ao respectivo assunto, este estudo possui como objetivo evidenciar os principais aspectos da visita domiciliar em saúde bucal na atenção primária através de uma revisão de literatura por meio da leitura de artigos nacionais e internacionais.

\section{REVISÃO BIBLIOGRÁFICA}

\section{Requisitos para um planejamento de atenção domiciliar}

Antes de ser executado uma atividade domiciliar, é necessário um planejamento muito claro do que a equipe de saúde que fazer nas residências, podendo ser desde uma visita só de cotidiano, como também uma visita de planejamento dos quais os profissionais irão estabelecer as condições, para eventuais tratamentos. Existem inúmeras vantagens na visita domiciliar pois estabelece uma aproximação maior com o território que está sendo cuidado, com isso fica mais fácil de elaborar um plano de tratamento que são centrados nas pessoas que de fato necessitam, e esses cuidados são relatados para os familiares, tendo tendência maior na resolutividade da atenção primária nas questões que envolvem a saúde bucal (SALAS MM, et al., 2015).

A incorporação do atendimento domiciliar aponta para uma restruturação e reorganização das práticas de saúde para além dos muros dos serviços de saúde, deslocando o olhar crítico para um espaço em que o paciente vive sendo enquadrado nesse aspecto, tanto o usuário, quanto a família e a comunidade nos quais as práticas das atenções estão enraizadas, estando diretamente relacionado com a mudança do serviço de atuação, ou seja, o serviço ofertado vai passar de um mais receptivo para ser um atendimento mais pró ativo, tentando identificar as particularidades, e identificar quais seriam aquelas famílias que de alguma forma precisam de um maior acesso a porta de entrada no SUS, porém de alguma forma não estão conseguindo chegar as unidades de Atenção Básica (SCHERER Cl e SCHERER MDA, 2015).

\section{Atenção as famílias}

A atenção às famílias e à comunidade é o objetivo central da visita domiciliar, sendo entendidas, famílias e comunidade, como entidades influenciadoras no processo de adoecer dos indivíduos os quais são regidos pelas relações com o meio e com as pessoas. E nesse caso o "sair para comunidade" precisa ter impacto na maneira de atuação dos profissionais, questionando seus conceitos acerca do modo de vivência e sobrevivência das famílias, essa atividade deve ser englobada por toda equipe multiprofissional, sendo fundamental para que tenha um bom trabalho com as famílias os agentes comunitários de saúde, porque são eles que vão poder também identificar quais situações vivem determinadas famílias (SILVA RM, 2016; SILVA $\mathrm{RM}$, et al., 2018).

\section{Subdivisões do AD}

A AD de nível 1, é destinada a pessoas que tenham uma dificuldade ou impossibilidade de se locomoverem até as unidades básicas de saúde até um serviço de saúde como um todo e que esteja dentro da capacidade de atenção das equipes que fazem a AD1, que são exatamente as equipes de saúde da família e as equipes de atenção domiciliar, a AD2 é destinada a usuários que demanda uma maior complexidade com maior frequência de cuidados que ultrapassa a capacidade de atuação da equipe de saúde da família e que além disso eles estejam em algumas situações que são previstas por essa portaria 2527 de 2011, quais sejam a necessidade de adaptação, curativos complexos e drenagem de abscesso, dependência de monitoramento frequente de sinais vitais ((BRASIL, 2013).

Levando em consideração as necessidades frequentes de exames de laboratório de menor complexidade, adaptação do paciente ou cuidador ao uso do dispositivo de traqueostomia, adaptação do uso de próteses, sondas e ostomias e acompanhamento domiciliar em pós-operatório, reabilitação de pessoas com deficiência permanente ou transitória, até apresentarem condições de frequentarem outros serviços de reabilitação, uso 
de aspirador de vias aéreas para higiene brônquica, acompanhamento de ganho ponderal de recém-nascidos de baixo peso, atenção nutricional permanente ou transitória, cuidados paliativos e necessidade de medicação endovenosa, muscular ou subcutânea, por tempo pré-estabelecido (BRASIL, 2013; SILVA RM, 2016).

Já os cuidados a AD3, além de todos os elementos que definem a essa necessidade de cuidados mais intensos, necessita da presença de uma maior complexidade tecnológica, já que na AD3 trabalha-se com a logística de oxigenoterapia com ventilação mecânica, diálise peritoneal, paracentese, sou seja, existem procedimentos que são realizados no domicílio e que define a AD3 (BRASIL, 2013; SILVA RM, 2016).

\section{Implantação do AD nas equipes de AP}

A Equipe Multiprofissional de Atenção Domiciliar (EMAD), vai se responsabilizar em geral por pacientes que são crônicos, em que encontram-se em condições angustiantes por um tempo determinado, em geral quem faz os cuidados em pacientes com situações crônicas vai ser a $A D$ de nível 1, composta pela equipe da saúde da família e equipe de atenção primária, sendo de suma importância ressaltar que a AD promovida pelas equipes interdisciplinares ou seja o Serviço de Atenção Domiciliar (SAD), vai ser complementar ou substitutivo (SILVA RM, 2016).

Com isso observa-se que ele irá substituir por exemplo o cuidar no hospital ou cuidar daquele paciente que está na Unidade de Pronto Atendimento (UPA), esperando uma vaga no hospital ou na unidade de pronto atendimento, mas que têm condições de ser tratado em sua residência, como é o caso de tratamentos com antibioticoterapia, tratamento por via intravenosa ou tratamento de uma ferida, curativo um pouco mais complexo, mas ela é complementar ao trabalho da $A B$, sendo por sua vez responsabilidade da equipe multiprofissional de estão presentes na $A B$ da equipe da saúde da família (SILVA RM, 2016; ANDRÉ PL, et al., 2019).

Porém é necessário lembrar que essas medidas não vêm a substituir os cuidados da atenção básica e exatamente por isso que os profissionais de saúde devem saber que a AD de nível 1 é o primeiro passo desse processo de cuidado, em espiral e que em uma série de situações a atenção básica ou será a única alternativa ou a melhor para o cuidado a essas pessoas (ROCHA NSM, et al., 2012; BRAÚNA APVS, et al., 2015).

\section{Planejamento}

Ao ser mensurado um planejamento para a organização do $A D$, deve-se levar em consideração a abrangência de medidas educativas não apenas como incrementações de estágios separados e consecutivos. Na conjuntura integral, as principais fases de uma otimização no planejamento das ações das quais devem ser as compreensões sobre a autenticidade, disposições dos obstáculos que lá existem e definição de metodologias utilizadas buscando o excelente efeito, elaboração de planejamentos na forma de atuar tendo em vista as atividades delineadas; assistencialismo e apreço (PINTO VG, 2013; SILVA RM, 2016).

A AD é composta por um conjunto de condutas estabelecidas nos trabalhos dos quais proporcionam como sendo um dos focos principais a promoção da saúde, possuindo como consequências esperadas precautelar e cuidar possíveis patologias com a ótica de restabelecer a recapacitação do usuário no AD, procurando asseverar o processo continuo dos cuidados, além disto fazer parte dos componentes na incorporação das redes de atenção à saúde (SALAS MM, et al., 2015). Como os demais membros que trabalham da área da saúde, o CD no campo da estratégia da saúde da família dispõe de sua transcendente relevância, como são os casos de serem capacitados em executar visitas domiciliares com intuito de oferecer prestabilidade de saúde oral, podendo ser elas de maneira individual ou coletiva (SILVA RM, 2016; ANDRÉ PL, et al., 2019).

A logística de obter uma atenção de maneira planejada na $A D$ visa conceber o processo de desospitalização na atenção básica, determinando obter conectividades de maneira mais aproximada entre os pacientes e os profissionais, tornando-se sinalizada como um dos componentes de transformações somatizadas e relativas as assistências à saúde, dado que as funcionalidades ocupacionais relacionados aos serviços oferecidos na saúde são oferecidos para toda conjectura familiar em suas respectivas residências, tendo como finalidade determinar ou reconstituir a saúde; elevando ao máximo o nível de independência; minimizando os segmentos de incapacidade ou até mesmo mal-estar provocado por determinada doença, buscando abranger sempre integrar aqueles pacientes que não apresentam melhoria da saúde (SILVA RM, et al., 2018). 
Em alguns casos, o gestor ou mesmo as equipes no processo de implantação ainda não possui o conhecimento da realidade que envolve o território pelo qual aquela equipe é responsável e isso é cada vez menos frequente, mas nessas situações os profissionais terão que trabalhar com elementos de estimativa da população, verificando dentro dessa estatística populacional a frequência e prevalência de doenças crônicas e os extremos da vida ( que leva em consideração a infância dos dois primeiros anos, onde encontra-se as doenças de traumatismos e síndromes genéticas metabólicas e logo em seguida a faixa etária que engloba os idosos, onde as doenças crônicas e degenerativas vão ser responsáveis pelo grande número de pessoas com perda de sua capacidade funcional) (MATOS GCM, et al., 2014; MIRANDA AF, et al., 2018).

Na maioria dos territórios existem equipes de Atenção Primária, seja elas de Atenção Básica de Saúde da Família que já conhecem a sua população, sabem as condições presentes no respectivo território, já são cadastrados os usuários que precisam de determinadas condições especiais seja pela antiga SAD, ou até mesmo pelo e-SUS Atenção Primária (e-SUS APS), então todos os elementos necessários para o planejamento são dados, esses elementos então podem serem usados pela equipe multidisciplinar para poderem executar um planejamento que envolve em primeiro lugar, a carda e demanda assistencial que essa população vai exigir em termos de cuidados no domicílio e por outro lado analisar a capacidade que essas equipes vão ter de suprir com as eventuais demandas (BIZERRIL DO, et al., 2015).

Para isso os profissionais abrem mão de uma série de ferramentas de instrumentos de escalas que vão estratificar as necessidades, seja através da vulnerabilidade de risco familiar, ou através da vulnerabilidade do risco individual, então deve-se lançar mão das escalas para que consiga organizar melhor o cuidado e poder planejar o cuidado domiciliar na AD de nível 1 (MACIEL JAC, et al., 2016).

\section{Atenção multiprofissional e o papel do CD aos pacientes que precisam de cuidados domiciliares}

A equipe multiprofissional deve ser unida para conseguir reconhecer como o indivíduo encontra-se, sendo de suma importância saber como os outros profissionais em cada área especifica está agindo, o CD deve cumprir com seu papel, tendo atenção na cavidade oral do paciente, porém deve ficar ciente sobre possíveis doenças sistêmicas que o paciente possua, buscando sempre dispor de uma visão integral (REIS WG, et al., 2015; MIRANDA AF, et al., 2018).

Silva RM, et al. (2018) destacam que os aspectos que envolvem os planejamentos para atendimento domiciliar devem dispor de um conceito amplo, tendo em numeras vezes características voltadas para a área político-administrativa, sendo ela no âmbito público ou privado. Entretanto mesmo sabendo que a declaração de elaboração de um plano detenha inúmeras maneiras de compreensão, entende-se como sendo uma maneira de fundamentar ações sociais por intermédio de elucidar possibilidades do desenvolvimento humanizado, pretendendo solucionar impasses e entender às indispensabilidades coletivas ou até mesmo individuais de determinada população.

Rocha DA e Miranda AF (2013) salientaram que os AD's apresentam metodologias benéficas para os pacientes atendidos, porém, para que esses resultados expressem bons frutos é necessário a obtenção de uma boa anamnese para só assim executar um planejamento do que irá ser realizado, esta forma de abordagem faz com quer os CD's detenham resultados benéficos, visto que essa conduta promove um atendimento diferenciado, atendendo de uma melhor maneira os usuários que necessitam de um processo terapêutico de maneira particularizada, como pode ser citado os pacientes que apresentam pouca ou nenhuma mobilidade, diferentes síndromes e pacientes idosos. Esses grupos característicos, a assistência odontológica apresenta várias dificuldades, devido as próprias circunstâncias pré-existentes, transcorrida desde problemas na cavidade bucal ou até mesmo a falta de conhecimento dos acompanhantes ou familiares.

Scherer Cl e Scherer MDA (2015) dizem que a elaboração de uma padronização de um plano adequado para essa forma de assistência apresenta divergentes tipos de barreiras, seja ela por causa da localização do usuário, a grande ocorrência de violência em alguns locais específicos da comunidade, a segregação das zonas periféricas de grandes metrópoles, escassezes de profissionais capacitados, agentes comunitários que não comunicam a vivência do paciente, dentre outros. 


\section{Visita Domiciliar em saúde bucal}

A visita domiciliar respeita e atende aos princípios norteadores das ações especificas nas diretrizes da Política nacional de Saúde Bucal, bem como os princípios da ética da saúde, o acesso universal para a assistência e o acolhimento, considerando o usuário em sua integralidade biopsicossocial, a criação de vínculo entre os profissionais da odontologia e os usuários, além da responsabilidade profissional, garantindo respostas resolutivas aos problemas do usuário. Podendo ser analisado que sempre é buscado trabalhar com a realidade das questões bucais de uma forma mais vinculada à realidade daquela família (NEVES M, et al., 2017).

Uma das questões que os CD's devem levar em consideração é deixar o paciente ciente de todas as informações e dos planos que irão ser executados, embora a visita domiciliar em saúde bucal não esteja limitada a um único perfil de usuários, devendo-se considerar o pressuposto da equidade das ações em saúde. Então muitas vezes alguns usuários que por motivo sejam eles físicos ou questões sociais e as vezes pela própria divisão do território ou questão de vulnerabilidade diárias com maior dificuldade de acesso, os CD's devem possuir um olhar um pouco mais ampliado para conseguir ofertar acesso para aquelas pessoas, buscando sempre fazer uma parceria com os agentes de saúde na identificação de usuários que necessitam dos cuidados odontológicos um pouco mais particularizado (SILVESTRE JAC, et al., 2013; BRAÚNA APVS, et al., 2015).

$\mathrm{Na}$ maioria dos casos os profissionais da odontologia cuidam dos usuários acamados, pacientes com dificuldade de acesso à unidade, por questões físicas, geográficas e sociais, famílias em área de risco e programas de vigilância, para os programas de vigilância em especifico é necessário ter um olhar crítico nas gestantes e em crianças menores de um ano. Por isso a conversa prévia muitas vezes procede para poder tentar identificar quem são as pessoas e formar um vínculo entre profissional e usuário (BIZERRIL DO, et al., 2015).

\section{CONSIDERAÇÕES FINAIS}

A perspectiva do atendimento domiciliar nos dias atuais ainda não são implantadas em todos os territórios no sentido de incorporação das atividades e estratégias de saúde bucal na saúde da família, no entanto vale ressaltar que o $C D$ e toda a equipe de saúde bucal devem serem inseri-las na rotina para a formação de uma conexão mais palpável entre os profissionais de saúde bucal e os usuários, apresentando como decorrência uma maior estrutura humanizada, não só da atenção, como também dos cuidados em saúde bucal. $O A D$ assegura a igualdade para todos e uma assistência igualitária a uma parte da sociedade afastada da odontologia tradicional demonstrando suas particularidades, prezando sempre pelo bem-estar físico e social dos pacientes através de atividades direcionadas para promoção e prevenção.

\section{REFERÊNCIAS}

1. ANDRÉ PL, et al. Atenção domiciliar em saúde bucal: experiência de integração ensino-serviço-comunidade em centro de saúde da família, SANARE, 2019; 18, (01): 90-97.

2. BIZERRIL DO, et al. Papel do cirurgião-dentista nas visitas domiciliares: atenção em saúde bucal. Rev Bras Med Fam Comunidade. 2015; 10(37): 1-8.

3. BRASIL. Ministério da Saúde. Portaria $\cong 963$ de 27 de maio de 2013. Redefine a Atenção Domiciliar no âmbito do Sistema Único de Saúde (SUS). Diário Oficial da União 2013.

4. BRASIL. Ministério da Saúde. Secretaria de Atenção à Saúde. Departamento de Atenção Básica. Caderno de Atenção Básica, no 17. Brasília: Ministério da Saúde; 2006.

5. BRAÚNA APVS, et al. Protocolo de visita domiciliar em saúde bucal. Prefeitura de Belo Horizonte. 2015; p. 1-10.

6. FERRAZ GA, LEITE ISG. Instrumentos de visita domiciliar: abordagem da odontologia na estratégia saúde da família. Rev APS 2016; 19(2):302-314.

7. MACIEL JAC, et al. Quando a saúde bucal bate à porta: protocolo para a atenção domiciliar em odontologia. Rev Bras Promoç Saude 2016; 29(4): 614-620.

8. MATOS GCM, et al. A inclusão da equipe de saúde bucal na Estratégia Saúde da Família: entraves, avanços e desafios. Ciênc Saúde Coletiva. 2014; 19(2): 373-82.

9. MIRANDA AF, et al. A necessidade da odontologia domiciliar e cuidados bucais em idosos dependentes. RCO, 2018; 2, (2): 33-38. 
10. NEVES M, et al. Atenção primária à saúde bucal no Brasil: processo de trabalho das equipes de saúde bucal. Cien Saude Colet 2017; 24(5): 1809-1820.

11. PINTO VG. Planejamento. In: Saúde Bucal Coletiva. São Paulo: Editora Santos, 6. ed. Cap. 2. 2013, p. 8-30.

12. REIS WG, et al. O trabalho do Cirurgião-Dentista na Atenção Primária à Saúde: entre o prescrito e o real. Saude Debate 2015; 39(104): 56-64.

13. ROCHA DA, FRANCO MA. Atendimento odontológico domiciliar aos idosos: uma necessidade na prática multidisciplinar em saúde: revisão de literatura. Rev Bras Geriatr Gerontol. 2013; 16(1): 181-9.

14. ROCHA DA, MIRANDA AF. Atendimento odontológico domiciliar aos idosos: uma necessidade na prática multidisciplinar em saúde: revisão de literatura Rev. Bras. Geriatr. Gerontol. 2013; 16(1):181-189.

15. ROCHA NSM, et al. Saúde bucal do idoso atendido na Policlínica Geral do Rio de Janeiro: uma análise radiográfica. Rev. Bras. de Odontol. 2012;69(2):238-41

16. SALAS MM, et al. Estimated prevalence of erosive tooth wear in permanent teeth of children and adolescents: an epidemiological systematic review and metaregression analysis. J Dent. 2015; 43(1): 42-50.

17. SCHERER CI, SCHERER MDA. Avanços e desafios da saúde bucal após uma década de Programa Brasil Sorridente. Rev Saúde Publica 2015; 49: 98.

18. SILVA RM, et al. Atuação da equipe de saúde bucal na atenção domiciliar na estratégia saúde da família: uma revisão integrativa. Rev. Ciência \& saúde 2018.

19. SILVA RM. Atenção à saúde bucal no domicílio no contexto da Estratégia Saúde da Família: reflexões a partir de uma revisão integrativa da literatura [monografia]. Florianópolis (SC): Universidade Federal de Santa Catarina; 2016.

20. SILVESTRE JAC, et al. Do Brasil em dentes ao Brasil sorridente: um resgate histórico das políticas públicas de saúde bucal no Brasil. Cadernos ESP 2013; 7(2): 28-39. 